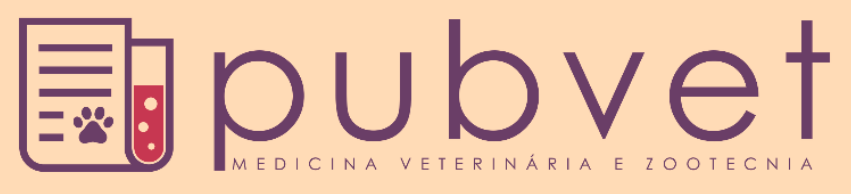

https://doi.org/10.31533/pubvet.v12n9a164.1-6

\title{
Técnica peridural com uso de lidocaína e bupivacaína associados ao fentanil, em cadelas submetidas à ovariosalpingohisterectomia
}

\author{
Catarina Rafaela Alves da Silva ${ }^{* *}$, Francisco Lima Silva ${ }^{2 \oplus}$, Francisco Solano Feitosa Júnior ${ }^{3 \oplus}$ \\ ${ }^{1}$ Residente Anestesiologia veterinária. Universidade Federal do Piauí. *Autor correspondência. E-mail: catarinarafaela@hotmail.com \\ ${ }^{I}$ Professor Universidade Federal do Piauí - Departamento de Clínica e Cirurgia Veterinária. E-mail: flimavet@hotmail.com \\ ${ }^{2}$ Professor Universidade Federal do Piauí-Departamento de Clínica e Cirurgia Veterinária. E-mail: solanofeitosa@bol.com.br
}

RESUMO. O crescente interesse pela anestesiologia veterinária observada nos últimos anos é fruto do reconhecimento dessa especialidade como fundamental ao exercício da medicina veterinária, haja visto que quanto mais amplo for o conhecimento acerca das técnicas anestésicas existentes, mais acertada será a escolha a ser feita. Objetivou-se com esta pesquisa comparar os efeitos provocados por dois protocolos anestésicos em cadelas submetidas à ovariossalpingohisterectomia, tendo como pré-medicação a acepromazina e tramadol, midazolam e propofol como agente indutor anestésico juntamente com associações via epidural com lidocaína-fentanil e bupivacaína-fentanil, a fim de observar os efeitos causados quanto as variáveis fisiológicas, duração e qualidade da anestesia e para tal, foram utilizadas 15 cadelas SRD, com peso médio 12,26 kg, hígidas, provenientes de abrigos que foram alocadas em três grupos de acordo com o protocolo a ser aplicado. Foram avaliados a frequência cardíaca, a frequência respiratória e a temperatura corpórea; bem como a dose média de anestésicos, reflexo sensitivo, motor e anal. Os valores de frequência cardíaca, embora se apresentem ligeiramente alterados, se mantiveram dentro dos parâmetros fisiológicos normais para a espécie canina. A frequência respiratória no grupo fentanil-lidocaína teve variação significativa em relação aos valores basais. Foi registrada hipotermia discreta nos animais de todos os grupos, de modo mais severo no grupo fentanilbupivacaína. O reflexo motor e sensitivo no grupo fentanil-bupivacaína ocorreu de forma mais retardada que no grupo fentanil-lidocaína além disso alguns animais do grupo fentanil-lidocaína apresentaram efeitos adversos tais como salivação e relaxamento do esfíncter anal a ponto de defecar. Conclui-se, portanto, que a técnica que utiliza o fentanil associado à bupivacaína é mais recomendável para a cirurgia de ovariossalpingohisterectomia, pois apresenta maior bloqueio nociceptivo, maior relaxamento e em consequência disso resulta em maior conforto para o animal durante $o$ trans e o pós-operatório.

Palavras chave: anestesia, bloqueio local, epidural, neuroeixo, protocolos

\section{Epidural technique with use of lidocaine and bupivacaine associated with fentanyl in bitches submitted to ovarian oysterectomy}

ABSTRACT. The increasing interest in veterinary anesthesiology observed in recent years
is the result of the recognition of this specialty as fundamental to the practice of veterinary
medicine, since the more extensive the knowledge about existing anesthetic techniques, the
better the choice to be made. The objective of this study was to compare the effects of two
anesthetic protocols on bitches submitted to ovarian oysterectomy, with acepromazine and
tramadol, midazolam and propofol as an anesthetic inducing agent together with epidural
combinations with lidocaine-fentanyl and bupivacaine-fentanyl, in order to observe the
effects caused by the physiological variables, duration and quality of the anesthesia, 15 
undefined (SRD) bitches with a mean weight of 12.26, healthy, were used from shelters that were allocated in three groups according to the protocol to be applied. Heart rate, respiratory rate and body temperature were evaluated; as well as the average dose of anesthetics, sensory, motor and anal reflex. The heart rate values, although slightly altered, remained within the normal physiological parameters for the canine species. The respiratory rate in the fentanyl-lidocaine group had a significant change from baseline. Discrete hypothermia was recorded in animals of all groups, more severely in the fentanylbupivacaine group. The motor and sensory reflex in the fentanyl-bupivacaine group occurred more delayed than in the fentanyl-lidocaine group and that some animals in the fentanyl lidocaine group had adverse effects such as salivation and relaxation of the anal sphincter to the point of defecation. Therefore, it is concluded that the technique that uses fentanyl associated with bupivacaine is more advisable for ovarian resection surgery, because it has a greater nociceptive block, greater relaxation and consequently results in greater comfort for the animal during trans and post-surgery.

Keywords: anesthesia, local block, epidural, neuraxis, protocols

\section{Técnica peridural con uso de lidocaína y bupivacaína asociados al fentanil, en perras sometidas a ovariohisterectomía}

RESUMEN. El creciente interés por la anestesiología veterinaria observada en los últimos años es fruto del reconocimiento de esa especialidad como fundamental al ejercicio de la medicina veterinaria, ya que cuanto más amplio sea el conocimiento acerca de las técnicas anestésicas existentes, más acertada será la elección a ser hecha. Se objetivó con esta investigación comparar los efectos provocados por dos protocolos anestésicos en perras sometidas a la ovariohisterectomía, teniendo como premedicación a acepromazina y tramadol, midazolam y propofol como agente inductor anestésico junto con asociaciones vía epidural con lidocaína-fentanil y bupivacaína-fentanil, a fin de observar los efectos causados en cuanto a las variables fisiológicas, duración y calidad de la anestesia y para ello, se utilizaron 15 hembras caninas criollas, con peso medio $12,26 \mathrm{Kg}$, sanas, provenientes de abrigos que fueron asignados en tres grupos de acuerdo con el protocolo a ser aplicado. Se evaluó la frecuencia cardíaca, la frecuencia respiratoria y la temperatura corporal; así como la dosis media de anestésicos, reflejo sensitivo, motor y anal. Los valores de frecuencia cardiaca, aunque se presentaron ligeramente alterados, se mantuvieron dentro de los parámetros fisiológicos normales para la especie canina. La frecuencia respiratoria en el grupo fentanil-lidocaína tuvo una variación significativa en relación a los valores basales. Se registró hipotermia discreta en los animales de todos los grupos, de modo más severo en el grupo fentanil-bupivacaína. El reflejo motor y sensitivo en el grupo fentanil-bupivacaína ocurrió de forma más retardada que en el grupo fentanillidocaína. Algunos animales del grupo fentanil-lidocaína presentaron efectos adversos tales como salivación y relajación del esfínter anal a punto de defecar. Se concluye, por lo tanto, que la técnica que utiliza el fentanil asociado a la bupivacaína es más recomendable para la cirugía de ovariohisterectomía, pues presenta mayor bloqueo nociceptivo, mayor relajación y en consecuencia de ello resulta en mayor conforto para el animal durante el trans y el posoperatorio.

Palabras clave: anestesia, bloqueo local, epidural, neuroaxial, protocolos

\section{Introdução}

O crescente interesse pela anestesiologia veterinária observada nos últimos anos é fruto do reconhecimento dessa especialidade como fundamental ao exercício da medicina veterinária (Fantoni, 2009). Para que sejam minimizados os riscos, devem ser feitas análises criteriosas na utilização desses fármacos, principalmente no que tange aos estudos A escolha da técnica anestésica apropriada e que cause mínimos efeitos adversos é muito importante. A anestesia por via peridural é considerada por diversos autores um procedimento extremamente seguro e que quando realizado de maneira adequada, possibilita a 
realização das mais diversas intervenções cirúrgicas.

Esta via, embora muito empregada na medicina veterinária, é utilizada quase que exclusivamente com os anestésicos locais associados à agente vasoconstritor. No entanto, estudos no homem demonstram que a eficácia dos anestésicos locais pode ser aumentada com a utilização de um opioide (Mourisse et al., 1992). O uso de opioides pela via peridural, no entanto, pode estar associado com efeitos adversos como depressão respiratória, excessiva sedação, vômitos tardios, retenção urinária e prurido (Wood et al., 1994). Entretanto, como a associação de anestésico local com opioide resulta em efeito sinérgico, a dose e os efeitos dose-permanente de ambos os fármacos podem ser reduzidos (Ackerman et al., 1998).

Dentre as inúmeras vantagens da anestesia regional, pode-se citar: o menor índice de mortalidade e morbidade quando comparado ao da anestesia geral, a necessidade de intubação orotraqueal é praticamente inexistente (Urban \& Urquhart, 1994); há analgesia pós-operatória; redução do estresse cirúrgico através da eliminação de estímulos dolorosos aferentes do local da operação, além do bloqueio dos nervos simpáticos eferentes até as glândulas endócrinas e metabólicas que são vistas após os atos cirúrgicos (Fragata \& Imagawa, 2008). O uso de opioides pela via peridural em pequenos animais, é de primordial importância. Desta forma, pretende-se obter mais uma modalidade de anestesia e de analgesia proporcionando maior conforto aos pacientes.

O objetivo deste trabalho visa comparar os efeitos do fentanil associado à lidocaína e bupivacaína por via peridural para proporcionar anestesia aos cães avaliando quantitativamente a eficácia anestésica, confrontando as doses administradas nos protocolos trabalhados e verificando a ocorrência de efeitos adversos.

\section{Material e métodos}

Foram utilizados 15 cães SRD (Sem raça definida), fêmeas, hígidas, provenientes de abrigos de animais e que foram submetidos à cirurgia de ovariosalpingohisterectomia eletiva. Os animais foram examinados pré-cirúrgicamente para avaliação da sanidade dos mesmos.

No momento da admissão, foi feita a ficha de identificação na qual consta o nome do animal e proprietário, no caso a associação, e dados obtidos mediante a anamnese; em seguida os animais foram alocados em três grupos distintos e encaminhados ao centro cirúrgico. Vale ressaltar que todos os animais foram submetidos a jejum alimentar de 12 horas e hídrico de seis horas, antes da realização do experimento.

A associação que abriga os cães foi informada quanto à realização da pesquisa e lhes foi reservado o direito de participar ou não da mesma, conforme lhes era conveniente. $\mathrm{O}$ protocolo anestésico utilizado em todos os animais deste estudo constituiu-se inicialmente de aferição dos parâmetros fisiológicos (frequência cardíaca, frequência respiratória e temperatura retal) os quais foram repetidos a cada 15 minutos obedecendo às etapas da cirurgia e após seu término, que no total compreendeu o tempo máximo de 60 minutos. Em seguida, foi administrada medicação pré-anestésica (MPA) realizada com acepromazina na dosagem de 0,05 $\mathrm{mg} / \mathrm{kg}$, por via intramuscular (IM) e tramadol na dosagem de $4 \mathrm{mg} / \mathrm{kg}$, por via intramuscular, posteriormente, foi realizada a cateterização da veia cefálica, pela qual foi administrada fluidoterapia, mantida durante todo o procedimento cirúrgico, com solução de ringer lactato, na infusão de $10 \mathrm{ml} / \mathrm{kg} / \mathrm{h}$. Consecutivamente, foi realizada a tricotomia e antissepsia do espaço lombossacro e abdominal ventral (dependendo do grupo), a partir de então procedeu-se com indução anestésica com os fármacos propofol e midazolam na proporção de 5 $\mathrm{mg} / \mathrm{kg}$ e $0,2 \mathrm{mg} / \mathrm{kg}$, respectivamente. Após o posicionamento do animal (esfinge), foi efetuada a anestesia epidural e deu-se continuidade ao procedimento com a entubação orotraqueal e durante a intervenção cirúrgica os mesmos foram mantidos com isofluorano, em quantidade proporcional à CAM, promovendo a sedação leve do animal e garantindo seus reflexos protetores.

Os animais foram distribuídos de maneira aleatória em 3 grupos constituídos por 5 animais cada (Grupo I, Grupo II e Grupo III), onde:

Grupo I (FL): Recebeu lidocaína e fentanil associados, na proporção $5 \mathrm{mg} / \mathrm{kg}(=0,25 \mathrm{ml} / \mathrm{kg})$ e $0,002 \mathrm{mg} / \mathrm{kg}$, respectivamente, no espaço lombossacral (Figura 1);

Grupo II (FB): Recebeu bupivacaína e fentanil associados, na proporção de $5 \mathrm{mg} / \mathrm{kg} \quad(=0,25$ $\mathrm{ml} / \mathrm{kg}$ ) e $0,002 \mathrm{mg} / \mathrm{kg}$, respectivamente, no espaço lombossacral;

Grupo III (C): Não recebeu medicamento por via peridural; 


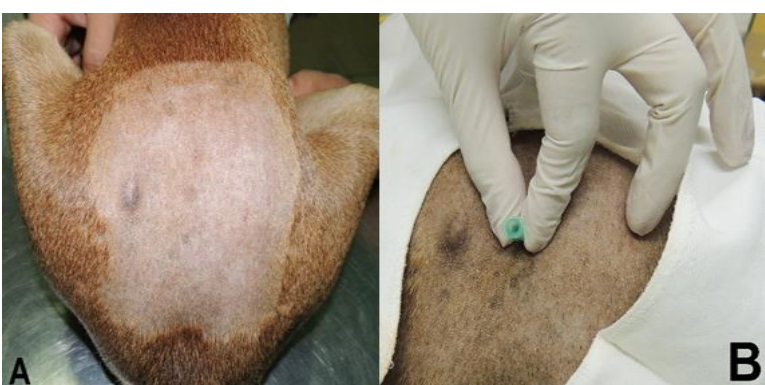

Figura 1. Tricotomia (A) e punção (B) do espaço lombossacro.

Nos animais constantes do Grupo I e II (FL e FB) foi realizada a tricotomia da região lombossacra e abdominal ventral seguida de anestesia peridural e já nos animais pertencentes ao GrupoIII (C), foi realizada somente a tricotomia abdominal ventral e não houve anestesia peridural. $\mathrm{O}$ procedimento transcorreu da seguinte forma:

Após serem tranquilizados com acepromazina $(0,05 \mathrm{mg} / \mathrm{Kg})$ e tramadol $(4 \mathrm{mg} / \mathrm{kg})$ e induzidos com propofol e midazolam, os animais foram posicionados em decúbito esternal com os membros posteriores flexionados cranialmente de modo que as cristas ilíacas puderam ser evidenciadas (posição de esfinge), identificando o espaço lombossacro através da palpação das cristas ilíacas com os dedos polegar e médio e com o indicador foi palpado o espaço lombossacro. Após a localização do espaço os animais foram submetidos à punção com agulha hipodérmica $22 \mathrm{G}$, o posicionamento correto da agulha foi confirmado através da prova da seringa de baixa resistência e então as drogas em estudo foram administradas.

O tratamento pós-operatório para atenuação da dor e da resposta inflamatória foi feito com meloxicam $(0,2 \mathrm{mg} / \mathrm{kg}, \mathrm{SC}$, nas primeiras $24 \mathrm{hs} \mathrm{e}$ a partir de então $0,1 \mathrm{mg} / \mathrm{kg}$, uma vez ao dia durante cinco dias), iniciado ao término da cirurgia. Os animais receberam antibioticoterapia com enrofloxacino com dose de ataque igual a 10 $\mathrm{mg} / \mathrm{kg}$ seguida de $5 \mathrm{mg} / \mathrm{kg}$, a cada 12 horas (BID) durante sete dias, e curativos locais da ferida cirúrgica, feitos diariamente, BID, com solução fisiológica, até a retirada dos pontos.

Foram avaliados a frequência cardíaca, a frequência respiratória e a temperatura corpórea; bem como a dose média de anestésicos, reflexo sensitivo, motor e anal. Foi utilizada estatística descritiva (média/desvio padrão).

\section{Resultados e discussão}

A dose média de propofol utilizada na indução foi de 7,4 $\pm 1,7$ no GC, 4,8 $\pm 3,1$ no GFL e 4,3 $\pm 1,1$ no GFB, sem variação estatística entre os grupos. Sobre a quantidade de isoflurano, foi utilizada na manutenção cirúrgica $6,8 \pm 1,2$ no $\mathrm{GC}, 7 \pm 2,0$ no GFL e 5,5 $\pm 2,7$ no GFB.

$\mathrm{O}$ reflexo motor (tentativa para levantar) e sensitivo (contração de esfíncter anal e reflexo interdigital), não variaram estatisticamente entre os grupos, tendo sido respectivamente $60 \pm 38,7$ minutos e $80 \pm 36,7$ minutos no GFL e $114 \pm 39,5$ minutos e $134 \pm 34,8$ no GFB.

A MPA utilizada, no caso a acepromazina, agiu dentro dos parâmetros estimados, pois de acordo com o relatado por Fantoni (2009) o efeito da mesma tem o período de latência em torno 5 a 10 minutos após administração intramuscular.

A dose de isofluorano utilizada no experimento foi menor do que a recomendada numa escala de até $50 \%$, se comparados os grupos fentanillidocaína e fentanil-bupivacaína em relação ao grupo controle, fato este que avigora o relato de McEwan et al. (1993) e Brunner et al. (1994) ao afirmarem que " o uso do fentanil promove diminuição da concentração alveolar mínima (CAM) do isofluorano em 50\%".

Quanto à frequência cardíaca, observou-se que era significativamente maior do GFL em relação aos demais grupos no momento T30, o qual apresentou redução em T60 em consonância com os demais (Tabela 1).

Tabela 1. Valores comparativos (média \pm desvio padrão) da frequência cardíaca (batimentos/minuto) entre caninos prémedicados com acepromazina e anestesiados com lidocaína e fentanil (GFL) ou bupivacaína e fentanil (GFB) associadas à anestesia peridural e aqueles que não receberam anestesia peridural (GC) quando submetidos à ovariosalpingohisterectomia.

\begin{tabular}{lcccc}
\hline \multirow{2}{*}{ Grupo } & \multicolumn{4}{c}{ Momentos } \\
\cline { 2 - 5 } & T-0 & T15 & T30 & T60 \\
\hline GFL & $92,8 \pm 18$ & $89,6 \pm 11$ & $137,4 \pm 68$ & $93,2 \pm 8$ \\
GFB & $84 \pm 18$ & $81,2 \pm 15$ & $98,6 \pm 19$ & $93,6 \pm 23$ \\
GC & $120 \pm 41$ & $76,8 \pm 16$ & $103 \pm 14$ & $98 \pm 39$ \\
\hline
\end{tabular}

Os valores de frequência cardíaca, embora se apresentem ligeiramente alterados no momento T30 do experimento, conforme previsto por Massone (2008) que diz que "os efeitos cardiovasculares indiretos (hipotensão, bradicardia) podem ocorrer após administração epidural ou espinhal, dependendo da extensão do 
bloqueio simpático concomitante"; se mantiveram dentro dos parâmetros fisiológicos normais para a espécie canina (Massone, 2008), ou seja, houve a preservação desde parâmetro em ambos os protocolos, durante todo o período experimental.

A frequência respiratória do GFB no momento T-30 apresentou um declínio considerável se comparado com os grupos GFL e GC, vindo a sofrer elevação a partir em T60 valor ainda menor que o grupo controle (GC). O GC e GFL apresentaram queda progressiva da frequência respiratória de T15 a T30 e partir de então, GC sofreu um aumento da frequência e GFL persistiu no declínio em T60 (Tabela 2).

Tabela 2. Valores comparativos (média \pm desvio padrão) da frequência respiratória (movimentos/minuto) entre caninos prémedicados com acepromazina e anestesiados com lidocaína e fentanil (GFL) ou bupivacaína e fentanil (GFB) associadas à anestesia peridural e aqueles que não receberam anestesia epidural (GC) quando submetidos à ovariosalpingohisterectomia.

\begin{tabular}{lcccc}
\hline \multirow{2}{*}{ Grupo } & \multicolumn{4}{c}{ Momentos } \\
\cline { 2 - 5 } & T-0 & T15 & T30 & T60 \\
\hline GFL & $60 \pm 10$ & $44,4 \pm 22$ & $29 \pm 12$ & $21,6 \pm 9$ \\
GFB & $41,6 \pm 5$ & $40,8 \pm 11$ & $7,2 \pm 3$ & $25,6 \pm 9$ \\
GC & $64,8 \pm 36$ & $43,6 \pm 18$ & $15,2 \pm 8$ & $40,8 \pm 18$ \\
\hline
\end{tabular}

A frequência respiratória no grupo fentanillidocaína teve variação significativa em relação aos valores basais (T60), o que corrobora a afirmação de Grond \& Radbruch (2006) que diz que "o efeito adverso mais grave associado a todos os opioides incluindo o fentanil, é a depressão respiratória". Esse achado pode estar associado ao fato de que no sistema respiratório, a acepromazina promove discreta diminuição da frequência (Muir III et al., 2001), mas potencializa a depressão causada por outros fármacos, no caso, o fentanil.

Acerca da temperatura corpórea não houve diferença significativa entre os grupos em todos os momentos avaliados, nem tampouco em cada grupo, ao longo do tempo (Tabela 3 ).

Nos grupos que foram utilizados peridural, foram visualizados relaxamento anal independente da associação anestésica local associada.

Foi registrada hipotermia discreta nos animais de todos os grupos, de modo mais severo no grupo fentanil-bupivacaína, a partir de T30 (momento da peridural), considerando os valores normais para a espécie que segundo Massone (2008) estão entre $38^{\circ} \mathrm{C}$ a $39^{\circ} \mathrm{C}$. Esse achado provavelmente deveu- se ao uso da acepromazina que tem efeito depressor e que, portanto produz queda da temperatura corporal, consequência da vasodilatação cutânea e da ação do fármaco nos mecanismos termorreguladores do hipotálamo (Spinosa et al.,1996), e que além disto pode ter potencializado o efeito do fentanil que também produz efeitos semelhantes. Outro fator a se pontuar neste aspecto é o fato de que a temperatura ambiente local, em média de $22^{\circ} \mathrm{C}$, pode ter interferido nesta perda de calor pelos pacientes e o colchão térmico utilizado não aqueceu os animais da maneira que deveria. Em face disto, é recomendável a utilização de medidas mais eficazes para o aquecimento passivo dos animais quando da utilização dos protocolos aqui estudados.

O reflexo motor e sensitivo no grupo fentanilbupivacaína ocorreu de forma mais retardada que no grupo fentanil-lidocaína. Tal achado revela que a primeira associação (fentanil-bupivacaína) demonstra resultados mais eficientes no quesito dor pós-operatória, o que confirma a afirmação de Massone (2008) que diz que "baixas concentrações podem ser usadas com vantagem para o alívio prolongado da dor, por exemplo, no parto ou no pós-operatório".

Tabela 3. Valores comparativos (média \pm desvio padrão) da temperatura corpórea entre caninos pré-medicados com acepromazina e anestesiados com lidocaína e fentanil (GFL) ou bupivacaína e fentanil (GFB) associadas à anestesia peridural e aqueles que não receberam anestesia epidural (GC) quando submetidos à ovariosalpingohisterectomia.

\begin{tabular}{lcccc}
\hline \multirow{2}{*}{ Grupo } & \multicolumn{4}{c}{ Momentos } \\
\cline { 2 - 5 } & T-0 & T15 & T30 & T60 \\
\hline GFL & $38,5 \pm 0,3$ & $38,7 \pm 0,5$ & $37,7 \pm 0,8$ & $36,4 \pm 0,8$ \\
GFB & $37,4 \pm 0,8$ & $38,2 \pm 0,4$ & $37,6 \pm 0,3$ & $36,3 \pm 0,5$ \\
GC & $38,1 \pm 0,08$ & $38,0 \pm 0,2$ & $37,4 \pm 0,6$ & $36,1 \pm 0,7$ \\
\hline
\end{tabular}

Ainda sobre o reflexo sensitivo, vale ressaltar que alguns animais do grupo fentanil-lidocaína apresentaram efeitos adversos tais como salivação e relaxamento do esfíncter anal a ponto de defecar, o que comprova a citação de Lumb \& Jones (1984) que diz que "alguns dos efeitos adversos observados após o uso do fentanil são agressividade pós-operatória, salivação e relaxamento do esfíncter anal, com ocasional defecação.

Com base no estudo realizado, foi possível inferir que o protocolo fentanil associado à bupivacaína em cirurgia de ovariossalpingohisterectomia apresentou maior 
bloqueio nociceptivo e em consequência disso resultou em maior conforto para o animal durante o trans e o pós-operatório.

\section{Referências bibliográficas}

Ackerman, B., Arwenstrom, E. \& Post, C. 1998. Local anesthesics pontiate spinal morphine antinocicepcion. Anesthesia \& Analgesia, 67, 943-948.

Brunner, M. D., Braithwaite, P., Jhaveri, R., McEwan A. I., Goodman D. K., Smith L. R. \& Glass P. S. 1994. MAC reduction of isofluorane by sufentanil. British Journal of Anesthesia, 72, 42-46.

Fantoni, D. T. 2009. Medicação Pré-Anestésica. In: Fantoni, D. T. \& Cortopassi, S. R. G. Anestesia em cães e gatos (cap. 13, p. 151158). Roca, São Paulo, Brasil.

Fragata, F. S. \& Imagawa, V. I. 2008. Analgesia na Terapia Intensiva. In: Santos, M. M. \& Fragata, F. S. Emergência e Terapia Intensiva Veterinária em Pequenos Animais: Bases para $o$ atendimento hospitalar (2a ed., cap. 41, p. 817-836). Roca, São Paulo, Brasil.

Grond, S. \& Radbruch, L. 2000. Clinical pharmacokinetics of transdermal opioids: focus on transdermal fentanyl. Clinical Pharmacokinetics, 38, 59-89.

Lumb, W. V. \& Jones, E. W. 1984. Veterinary anesthesia (2a ed., 693 p.). Lea \& Febiger, Philadelphia, US.

Massone, F. 2008. Anestesiologia Veterinária Farmacologia e Técnicas (5a ed., 572 p.). Guanabara Koogan, Rio de Janeiro, BR.
McEwan, A. I., Smith, C., Dyar, O., Goodman, D., Smith, L. R. \& Glass, P. S. 1993. Isofluorane minimum alveolar concentration reduction by fentanyl. Anesthesiology, 78, 864-869.

Mourisse, J., Hansebos, M. A. \& Gielen, M. J. 1992. Epidural bupivacaíne,sufentanil or the combination for post-thoracotomy pain. Acta Anaesthesiologica Scandinavica, 36, 70-4.

Muir III, W. W., Hubbell, J. A., Skarda, R. T. \& Bednarski, R. M. 2001. Manual de Anestesia Veterinária (3a ed., cap. 3, p. 31-44). Artmed Editora, Porto Alegre, BR.

Spinosa, H, S., Gorniak, S. L. \& Brenardi, M. M. 1996. Farmacologia aplicada à Medicina Veterinária (cap. 14, p.131-139). Guanabara Koogan, Rio de Janeiro, BR.

Wood, G. E., Goresky, G. V., Klassen, K. A., Kuwahara, B. \& Neil, S. G. 1994. Complications of continuous epidural infusions for post operative analgesia in children. Canadian Journal of Anesthesia, 41(7), 613-620.

Urban, M. K. \& Urquhart, B. 1994. Evaluation of brachial plexus anesthesia for upper extremity surgery. Regional Anesthesia, 19, 175-82.

Recebido: 15 Jun. 2018

Aprovado: 6 Jul. 2018

Publicado: 24 Ago., 2018

Licenciamento: Este artigo é publicado na modalidade Acesso Aberto sob a licença Creative Commons Atribuição 4.0 (CC-BY 4.0), a qual permite uso irrestrito, distribuição, reprodução em qualquer meio, desde que o autor e a fonte sejam devidamente creditados. 